\title{
Efficient Computation of Min and Max Sensor Values in Multihop Networks
}

\author{
Nuno Pereira ${ }^{1}$, Bjorn Andersson', Eduardo Tovar' ${ }^{1}$, Paulo Carvalho ${ }^{2}$ \\ Instituto Politécnico do Porto ${ }^{1}$ \\ Porto, Portugal \\ E-mail: $\{$ npereira,emt,bandersson $\}$ at dei.isep.ipp.pt \\ Universidade do Minho ${ }^{2}$ \\ Departamento de Informática \\ P-4710-057 Braga, Portugal \\ E-mail: pmc at di.uminho.pt
}

\begin{abstract}
Consider a wireless sensor network (WSN) where a broadcast from a sensor node does not reach all sensor nodes in the network; such networks are often called multihop networks. Sensor nodes take sensor readings but individual sensor readings are not very important. It is important however to compute aggregated quantities of these sensor readings. The minimum and maximum of all sensor readings at an instant are often interesting because they indicate abnormal behavior, for example if the maximum temperature is very high then it may be that a fire has broken out. We propose an algorithm for computing the min or max of sensor readings in a multihop network. This algorithm has the particularly interesting property of having a time complexity that does not depend on the number of sensor nodes; only the network diameter and the range of the value domain of sensor readings matter.
\end{abstract}

in the book "Intelligent Technical Systems", Lecture Notes on Electrical Engineering series, Vol.38, pp.233-246, Springer, 2009

(C) 2009 CCG I Last modified Sun Jul 05, 2009 by Paulo Carvalho 